\title{
Author Correction: Do no harm: a roadmap for responsible machine learning for health care
}

Jenna Wiens (D), Suchi Saria, Mark Sendak (D), Marzyeh Ghassemi, Vincent X. Liu, Finale Doshi-Velez, Kenneth Jung, Katherine Heller, David Kale, Mohammed Saeed, Pilar N. Ossorio, Sonoo Thadaney-Israni and Anna Goldenberg

Correction to: Nature Medicine https://doi.org/10.1038/s41591-019-0548-6, published online 19 August 2019.

In the version of this article initially published, an affiliation was missing for author Anna Goldenberg: Child and Brain Development Program, CIFAR, Toronto, Ontario, Canada. The error has been corrected in the HTML and PDF versions of the article.

\section{Publisher Correction: Enhancer signatures stratify and predict outcomes of non-functional pancreatic neuroendocrine tumors}

Paloma Cejas, Yotam Drier D, Koen M. A. Dreijerink, Lodewijk A. A. Brosens, Vikram Deshpande,

Charles B. Epstein (D, Elfi B. Conemans, Folkert H. M. Morsink, Mindy K. Graham, Gerlof D. Valk, Menno R. Vriens,

Carlos Fernandez-del Castillo, Cristina R. Ferrone, Tomer Adar, Michaela Bowden, Holly J. Whitton, Annacarolina Da Silva, Alba Font-Tello, Henry W. Long, Elizabeth Gaskell, Noam Shoresh, Christopher M. Heaphy (D), Ewa Sicinska,

Matthew H. Kulke, Daniel C. Chung, Bradley E. Bernstein (D) and Ramesh A. Shivdasani (D)

Correction to: Nature Medicine https://doi.org/10.1038/s41591-019-0493-4, published online 21 May 2019.

In the version of this article initially published, a numbering error mistakenly associated author Paloma Cejas with affiliation 15, Hebrew University; the affiliation footnote has been removed. Affiliations 10 (published as Massachusetts General Hospital and Harvard Medical School) and 11 (published as UMC Utrecht Cancer Center) were incorrectly transposed, affecting authors Menno R. Vriens, and Carlos Fernandez-del Castillo and Christina R. Ferrone, respectively. In Figure 2a, the image was misoriented by $90^{\circ}$, resulting in incorrect orientation of the dendrogram. The errors have been corrected in the HTML and PDF versions of the article. 